\title{
COVID-19: Frequência de casos reagentes em profissionais de saúde de um hospital
}

\section{referência no Paraná}

\author{
COVID-19: Frequency of positive cases in healthcare professionals at a reference hospital in \\ Paraná \\ COVID-19: La incidencia de casos positivos en profesionales de la salud en un hospital de \\ referencia no Paraná
}

\begin{abstract}
Resumo
O objetivo deste trabalho foi identificar o perfil e a frequência de casos rastreados de infecção pelo novo coronavírus (COVID-19) entre profissionais de saúde de uma unidade hospitalar referência no atendimento à doença. Trata-se de um estudo transversal em que foram utilizados os dados da Ficha de Notificação de Síndrome Respiratória COVID-19 e realizado os exames de RT-qPCR e Teste Rápido. Foram calculadas as frequências absolutas e relativas, usadas para descrever o perfil da amostra. Para comparação entre casos confirmados ou não da COVID-19, foi realizado o teste qui-quadrado com correção de continuidade de Yates, sendo considerados estatisticamente significativos os valores que apresentaram p <0,05. Foram testados 630 profissionais, com maior percentual de casos confirmados de profissionais do sexo feminino, idade abaixo de 40 anos, com atuação direta ao paciente, realização de pelo menos um teste para SARS-CoV-2 e maior frequência na hora da testagem dos sintomas de cefaleia, dor de garganta e mialgia. Do total, foram identificados 81 exames positivos. No teste de associação, identificou-se relação entre a sintomatologia e o número de sintomas dos casos reagentes para SARS-CoV-2 $(\mathrm{p}=0,001)$. Apesar de não significativo, destaca-se o número de profissionais do sexo feminino e que atuam no setor de clínicas com resultados reagentes. Monitorar e identificar os casos reagentes para o SARS-CoV-2 é essencial para auxiliar na prevenção da disseminação do vírus, bem como estabelecer mecanismos de prevenção e monitoramento da saúde do trabalhador do serviço de saúde.
\end{abstract}

Palavras-chave: Profissionais da saúde; COVID-19; SARS-CoV-2. 


\begin{abstract}
The aim of this study was to identify the profile and frequency of infections of the new coronavirus (COVID-19) among healthcare staff at reference hospital for treating the disease. This is a cross-sectional study that used data from COVID-19 Respiratory Syndrome Notification Records and the results obtained by RT-qPCR and Rapid Test. Absolute and relative frequencies were used to describe the sample. For comparisons between positive and negative cases, the chi-square test with Yates continuity correction was deployed, and were considered statistically significant the values that presented $p<0,05$. A total of 630 professionals were tested, and the highest percentage of confirmed cases was found among female professionals, aged below 40 years, with direct contact with COVID-19 patients, who performed at least one test for SARS-CoV-2; moreover, at the time of testing, headache, sore throat, and myalgia were the most reported symptoms. Eighty-one (81) positive tests were identified. A relationship between symptomology and the number of reagent cases to SARS-CoV-2 was found $(p=0.001)$. Although not significant, it is noteworthy the number of positive cases among female professionals and in those who work in the private clinics. In sum, monitoring and identifying positive SARS-CoV-2 is essential to assist prevention efforts against virus dissemination, as well as to establish safety routines for healthcare professionals.
\end{abstract}

Keywords: Healthcare Professional; COVID-19; SARS-CoV-2.

\title{
Resumen
}

El objetivo de este estudio fue identificar el perfil y la frecuencia de los casos de infección por el nuevo coronavirus (COVID-19), entre los profesionales de la salud de un hospital de referencia en la atención de la enfermedad. Estudio transversal en el que se utilizaron datos del Formulario de Notificación de Síndrome Respiratorio COVID-19, y se realizaron los exámenes RT-qPCR y Prueba Rápida. Se calcularon las frecuencias absolutas y relativas utilizadas para describir el perfil de la muestra. Para la comparación entre casos confirmados y no confirmados de COVID-19, se realizó la prueba de chi-cuadrado con corrección de continuidad, considerándose estadísticamente significativos los valores que presentaron $\mathrm{p}<0.05$. Se evaluaron un total de 630 profesionales, con el mayor porcentaje de casos confirmados de profesionales mujeres, menores de 40 años, que trabajaron directamente con el paciente, realización de al menos una prueba para el SARS-CoV-2 y con más frecuencia en el momento de la prueba para detectar síntomas de dolor de cabeza, dolor de garganta y mialgia. Del total de 81 pruebas positivas que se identificaron. La prueba de asociación identificó una relación entre la sintomatología y el número de síntomas de casos reactivos para el SARSCoV-2 ( $\mathrm{p}=0,001)$. Aunque no significativo, destaca el número de mujeres profesionales y que trabajan en el sector clínico con resultados reactivos. El seguimiento y identificación de casos reactivos al SARS-CoV-2 es esencial para ayudar a prevenir la propagación del virus, así como para establecer mecanismos de prevención y seguimiento de la salud de los trabajadores de los servicios de salud.

Palabras clave: Profesionales de la salud; COVID-19; SARS-CoV-2.

\section{Introdução}

Em 31 de dezembro de 2019, a China comunicou a descoberta de um novo tipo de coronavírus, após relatos de infecções respiratórias na província de Wuhan (Organização Mundial da Saúde, 2020a). Um mês após a declaração, a Organização Mundial da Saúde (OMS) anuncia o contágio como uma Emergência de Saúde Pública de importância Internacional, representando o mais alto nível de alerta da Organização, feito pela sexta vez na história (Organização Mundial da Saúde, 2020a). No dia 11 de fevereiro, a nova doença foi denominada de COVID-19, caracterizado como uma pandemia pela OMS no dia 11 de março de 2020 (Organização Mundial da Saúde, 2020a). No Brasil, o primeiro registro se deu no dia 26 de fevereiro (Ministério da Saúde, 2020a) e, desde então, os índices de contágio e de mortalidade vêm apresentando um crescimento exponencial em todo o país, de forma a sobrecarregar o Sistema Único de Saúde (SUS) e os hospitais particulares de modo nunca antes visto (Organização Mundial da Saúde, 2020). Até o presente momento, o número de contaminados passa dos 18 milhões, com mais de 500 mil mortes registradas (Ministério da Saúde, 2020a). Assim como nas pandemias que ocorreram no passado, os esforços iniciais estão direcionados a compreender os mecanismos fisiopatológicos e conter a disseminação do patógeno (Ornell et al., 2020; Shah et al., 2020).

De acordo com o Ministério da Saúde (Ministério da Saúde, 2020b), a COVID-19 é uma infecção respiratória aguda causada pelo novo coronavírus SARS-CoV-2, que é um betacoronavírus identificado em amostras de lavado broncoalveolar dos pacientes acometidos com uma pneumonia até então desconhecida. Por se tratar de um subgênero Sarbecovírus da família Coronaviridae, é conhecido como o sétimo coronavírus a infectar seres humanos.

O Ministério da Saúde destaca que a COVID-19 pode se apresentar nas formas mais leves - com sintomas como febre, 
cansaço e tosse seca -, que são a maioria dos casos (cerca de 80\%), mas também pode se desenvolver nas formas moderadas a grave, onde os pacientes precisam de atendimento hospitalar devido a dificuldade respiratória levando até uma insuficiência, precisando de intervenção multiprofissional qualificada. Alguns procedimentos médicos realizados durante a assistência aos pacientes com COVID-19 podem produzir gotículas muito pequenas (aerossóis), capazes de permanecer suspensas no ar por longos períodos. Os aerossóis podem ser inalados tanto pelos profissionais como por outras pessoas que estiverem nas unidades de saúde, caso as mesmas não estiverem usando o equipamento de proteção individual adequado e seguindo os protocolos de barreira biológica (Ministério da Saúde, 2020b).

Segundo o Centro de Medicina Baseada em Evidências da Universidade de Oxford, os profissionais de saúde apresentam maior risco de exposição e podem ser vetores da transmissão do vírus. Países que já passaram pela pandemia, como a China, relatam a contaminação de mais de 3.300 profissionais de saúde, o que corresponde a $4 \%$ das infecções relatadas (Adams \& Walls, 2020). Anelli et al., (2020) relatam que na Itália, até o mês de março, foi detectado 4.824 profissionais de saúde infectados com COVID-19 (9\% do total de casos), e ainda com 24 mortes de profissionais médicos, mostrando-se mais agravante do que as observadas na China (3.300 profissionais de saúde infectados), fazendo com que os italianos fechassem as portas dos hospitais por um período por causa da infecção que circulava entre médicos e enfermeiras . Já um estudo divulgado sobre a triagem por PCR de profissionais de saúde assintomáticos no hospital de Londres, que realizou testagem em profissionais durante uma semana, observou que as taxas de infecção assintomáticas entre os profissionais de saúde acompanharam a curva de infecção da população geral de Londres, atingindo um pico de 7,1\% e do total de casos da COVID-19 dos 44 profissionais de saúde que testaram reagente para SARS-CoV-2, 12 (27\%) não apresentaram sintomas na semana anterior ou posterior à positividade (Treibel et al., 2020).

Os profissionais de saúde, independente da categoria profissional, não apenas correm maior risco de infecção, como também podem ampliar os surtos nas unidades de saúde se ficarem doentes. Identificar e gerenciar profissionais de saúde que foram expostos a um paciente com COVID-19 é de grande importância na prevenção da transmissão de serviços de saúde e na proteção de funcionários e pacientes vulneráveis em ambientes de saúde.

Considerando a situação atual da pandemia no Brasil, o objetivo deste trabalho foi identificar a frequência de casos confirmados da COVID-19 entre profissionais de saúde do Hospital Regional do Sudoeste Walter Alberto Pecóits de Francisco Beltrão (HRSWAP), que se infectaram com o vírus do SARS-CoV-2, analisando o perfil profissional contaminado.

\section{Metodologia}

Trata-se de um estudo transversal, quantitativo, com coleta de dados numéricos onde foram aplicados métodos estatísticos (Pereira et al., 2018) para realizar o levantamento de dados durante os meses de março a novembro do ano de 2020 no Hospital Regional do Sudoeste Walter Alberto Pecóits de Francisco Beltrão (HRSWAP), Paraná. O HRSWAP é uma unidade terciária, de gestão estadual, que atende somente o Sistema Único de Saúde. É habilitada para atendimentos de média e alta complexidade. A instituição possui 108 leitos, oferecendo serviços nas especialidades de ortopedia, trauma, clínica cirúrgica, médica, obstetrícia, pediatria e psiquiatria. Encontra-se habilitada com uma Unidade de Terapia Intensiva Adulto (09 leitos), uma Unidade de Terapia Intensiva Neonatal (10 leitos) e uma Unidade de Cuidados Intermediários Neonatal Convencional (5 leitos). Frente a nova realidade do Novo Coronavírus a unidade tornou-se referência para o atendimento dos pacientes com suspeita e os casos confirmados da COVID-19 sendo disponibilizado para estes pacientes mais 10 leitos de UTI/adulto, 1 leito de UTI pediátrica e 10 leitos de clínica médica (HRSWAP, 2020).

Ao total, a unidade conta com 703 profissionais. Destes 45,3\% são profissionais da enfermagem (252 técnicos de enfermagem e 66 enfermeiros), $31,15 \%$ de outras categorias de profissionais da saúde que atuam no atendimento ao paciente e 23,62\% de pessoal de apoio à estrutura do hospital (HRSWAP, 2020). 
Os dados coletados foram retirados da Ficha de Notificação de Síndrome Respiratória COVID-19 do HRSWAP Profissionais de Saúde, com coleta de exame de RT-qPCR, analisando os dados sociodemográficos, categoria profissional, sinais e sintomas do Novo Coronavírus, comorbidades, histórico de viagens, contato. Nesse estudo foram incluídos todos os profissionais que atuam na unidade hospitalar que foram testados pela saúde ocupacional do HRSWAP. Para análise dos dados os profissionais foram agrupados segundo a função desempenhada $(n=40)$ e de acordo com o setor que trabalhavam $(\mathrm{n}=76)$. As funções foram divididas em 4 categorias de acordo com o tipo de trabalho desenvolvido dentro da instituição, sendo: assistência direta ao paciente, assistência indireta, administrativo e serviços de apoio. Os setores foram categorizados de acordo com o local de trabalho, sendo: os que trabalham com assistência aos pacientes com covid-19 (Unidade de Terapia Intensiva - UTI e clínicas), assistência aos pacientes críticos (UTI geral, UTI neonatal e Unidade de Cuidados Intermediários UCI), assistência aos pacientes nos demais setores do hospital e o serviço administrativo e de apoio. Em relação a idade média dos profissionais foi realizado o teste estatístico o qual foi encontrado a média de idade de 38,7 anos com um intervalo de $95 \%$ confiança.

Os dados coletados foram digitados na planilha do Microsoft Excel for Windows-7 (Microsoft Office 2007) e posteriormente explorados no pacote estatístico IBM SPSS (Statistical Package for the Social Sciences), versão 21. Foram calculadas as frequências absolutas (n) e relativas (\%) sendo usadas para descrever o perfil da amostra. O pressuposto de distribuição normal foi testado pelo teste de Kolmogorov-Smirnov. O teste qui-quadrado com correção de continuidade foi realizado para comparar os casos confirmados e não confirmados da presença do vírus SARS-CoV-2 das variáveis categóricas, sendo considerados estatisticamente significativos os valores que apresentaram $p<0,05$. Finalmente, salienta-se que o estudo respeitou os preceitos éticos, tendo sido aprovado no comitê de ética sob o número 4.112.388.

\section{Resultados}

As características da amostra são apresentadas na Tabela 1. Observa-se um maior percentual de profissionais do sexo feminino, com idade abaixo de 40 anos, com atuação direta ao paciente, com a realização de pelo menos um teste para SARSCoV-2, com maior frequência na hora da testagem dos sintomas de cefaleia, dor de garganta e mialgia. Ao total foram identificados 81 exames positivos para SARS-CoV-2, destes 03 profissionais foram testados por RT-qPCR e o teste rápido, testando positivo para ambos os testes. 
Tabela 1 - Caracterização dos profissionais que foram testados para a presença do vírus SARS-CoV-2 em uma unidade hospitalar referência para atendimento de pacientes COVID-19 no ano de 2020.

\begin{tabular}{|c|c|c|c|}
\hline Variáveis & $\mathrm{N}$ & $\%$ & IC95\% \\
\hline \multicolumn{4}{|l|}{ Sexo } \\
\hline Masculino & 478 & 75,9 & $72-79$ \\
\hline Feminino & 152 & 24,1 & $20-27$ \\
\hline \multicolumn{4}{|l|}{ Faixa etária } \\
\hline$>40$ anos & 370 & 58,8 & $54-62$ \\
\hline$\leq 40$ anos & 260 & 41,2 & $35-45$ \\
\hline \multicolumn{4}{|l|}{ Profissão } \\
\hline Médico & 28 & 5 & $3,5-7,3$ \\
\hline Enfermeiro & 49 & 9 & $6-11$ \\
\hline Técnico enfermagem & 183 & 33,5 & $29-37$ \\
\hline Outros & 287 & 52,5 & $48-56$ \\
\hline \multicolumn{4}{|c|}{ Assistência ao paciente } \\
\hline Direta & 352 & 55,9 & $52-59$ \\
\hline Indireta & 53 & 8,4 & $6-10$ \\
\hline Administrativo & 140 & 22,2 & $19-25$ \\
\hline Serviço de apoio & 85 & 13,5 & $10-16$ \\
\hline \multicolumn{4}{|l|}{ Local de atuação } \\
\hline COVID-19 & 81 & 12,9 & $10-15$ \\
\hline UTIs & 89 & 14,1 & $11-16$ \\
\hline Clínicas & 202 & 32 & $28-35$ \\
\hline Outros setores & 258 & 41 & $37-44$ \\
\hline \multicolumn{4}{|l|}{ Comorbidades } \\
\hline Sim & 57 & 9 & $6-11$ \\
\hline Não & 573 & 91 & $88-98$ \\
\hline \multicolumn{4}{|l|}{ Número de sintomas* } \\
\hline Nenhum & 464 & 73,8 & $70-77$ \\
\hline 1 a 3 & 86 & 13,7 & $11-16$ \\
\hline$\geq 4$ & 78 & 12,5 & $9-15$ \\
\hline \multicolumn{4}{|l|}{$\overline{\text { Sintomatologia }}$} \\
\hline Tosse & 71 & 11,3 & $08-13$ \\
\hline Cefaleia & 97 & 15,4 & $12-18$ \\
\hline Obstrução nasal & 24 & 3,8 & $2-5$ \\
\hline Coriza & 71 & 11,3 & $8-13$ \\
\hline Mialgia & 77 & 12,2 & $9-14$ \\
\hline Diarréia & 39 & 6,2 & $4-8$ \\
\hline Náusea & 33 & 5,2 & $3-7$ \\
\hline Calafrios & 28 & 4,4 & $2-6$ \\
\hline Adinamia & 27 & 4,3 & $2-5$ \\
\hline Febre & 32 & 5,1 & $3-6$ \\
\hline Vômito & 18 & 2,9 & $1-4$ \\
\hline Dor de garganta & 84 & 13,3 & $10-16$ \\
\hline \multicolumn{4}{|c|}{ Número de testes realizados } \\
\hline Um & 496 & 78,7 & $75,5-81,9$ \\
\hline Dois & 134 & 21,3 & $18,1-24,5$ \\
\hline \multicolumn{4}{|l|}{ Exame RT-qPCR } \\
\hline Realizado & 277 & 44 & $40,1-47,8$ \\
\hline Não realizado & 353 & 56 & $52,2-59,9$ \\
\hline \multicolumn{4}{|c|}{ Resultado Exame RT-qPCR } \\
\hline Reagente & 71 & 25,7 & $20,5-30,8$ \\
\hline Não reagente & 206 & 74,3 & $69,2-79,5$ \\
\hline \multicolumn{4}{|c|}{ Exame Teste Rápido (TR) } \\
\hline Realizado & 488 & 77,5 & $74,2-80,7$ \\
\hline Não realizado & 142 & 22,5 & $19,3-26,8$ \\
\hline \multicolumn{4}{|l|}{ Resultado Exame (TR) } \\
\hline Reagente & 13 & 2,7 & $1,2-4,1$ \\
\hline Não reagente & 475 & 97,3 & $95,9-98,8$ \\
\hline \multicolumn{4}{|c|}{ Resultados testes COVID-19 } \\
\hline Reagente & 81 & 12,9 & $10,2-15,5$ \\
\hline Não reagente & 544 & 87,1 & $84,4-89,6$ \\
\hline
\end{tabular}

Nota: * 02 participantes não foram registrados se havia ou não presença de sintomas. Fonte: Dados da Pesquisa.

No teste de associação identificou-se relação entre a sintomatologia e o número de sintomas dos casos reagentes para 
SARS-CoV-2 ( $<$ 0,001). Apesar de não significativo, destaca-se o número de profissionais do sexo feminino e que atuam no setor de clínicas com maior frequência de casos reagentes, conforme descrito na Tabela 2.

Tabela 2 - Distribuição por variáveis sociodemográficas, profissionais e sintomatológicas dos profissionais que foram testados para a presença do vírus SARS-CoV-2 em uma unidade hospitalar referência para atendimento de pacientes COVID-19 no ano de 2020.

\begin{tabular}{|c|c|c|c|c|c|}
\hline \multirow{3}{*}{ Variáveis } & \multicolumn{4}{|c|}{ SARS-CoV-2 } & \multirow{3}{*}{ p-value } \\
\hline & \multicolumn{2}{|c|}{ Não reagente } & \multicolumn{2}{|c|}{ Reagente } & \\
\hline & $\mathrm{n}$ & $\%$ & $\mathrm{n}$ & $\%$ & \\
\hline \multicolumn{6}{|l|}{ Sexo } \\
\hline Masculino & 127 & 23,2 & 25 & 30,9 & \multirow{2}{*}{0,171} \\
\hline Feminino & 421 & 78,8 & 56 & 69,1 & \\
\hline \multicolumn{6}{|l|}{ Idade } \\
\hline$>40$ anos & 292 & 53,4 & 41 & 50,6 & \multirow[t]{2}{*}{0,642} \\
\hline$\leq 40$ anos & 255 & 40,6 & 40 & 49,4 & \\
\hline \multicolumn{6}{|l|}{ Profissão } \\
\hline Médico & 28 & 5,1 & 07 & 8,6 & \multirow[t]{4}{*}{0,461} \\
\hline Enfermeiro & 49 & 9 & 09 & 11,1 & \\
\hline Técnico enfermagem & 183 & 33,5 & 28 & 34,6 & \\
\hline Outros & 287 & 52,5 & 37 & 45,7 & \\
\hline \multicolumn{6}{|l|}{ Assistência ao paciente } \\
\hline Direta & 302 & 55,1 & 49 & 60,5 & \multirow[t]{2}{*}{0,362} \\
\hline Indireta & 246 & 44,9 & 32 & 39,5 & \\
\hline \multicolumn{6}{|l|}{ Local de atuação } \\
\hline COVID-19 & 71 & 13 & 10 & 12,3 & \multirow[t]{4}{*}{0,184} \\
\hline UTI's & 76 & 13,9 & 13 & 16,1 & \\
\hline Clínicas & 168 & 30,7 & 33 & 40,7 & \\
\hline Outros setores & 233 & 42,5 & 25 & 30,9 & \\
\hline \multicolumn{6}{|l|}{ Comorbidades } \\
\hline Não & 499 & 91,1 & 73 & 90,1 & \multirow[t]{2}{*}{0,784} \\
\hline Sim & 49 & 8,9 & 08 & 9,9 & \\
\hline \multicolumn{6}{|l|}{ Sintomatologia } \\
\hline Não & 417 & 76,1 & 48 & 59,3 & \multirow[t]{2}{*}{0,001} \\
\hline Sim & 131 & 23,9 & 33 & 40,7 & \\
\hline \multicolumn{6}{|l|}{ Número de sintomas } \\
\hline Nenhum & 416 & 76,1 & 48 & 59,3 & \multirow[t]{3}{*}{0,001} \\
\hline 1 a 3 & 65 & 11,9 & 21 & 25,9 & \\
\hline$\geq 4$ & 66 & 12,1 & 12 & 14,8 & \\
\hline
\end{tabular}

Fonte: Dados da Pesquisa.

\section{Discussão}

A doença COVID-19 é uma infecção viral altamente transmissível que pode causar síndrome respiratória aguda grave 2 (SARS-CoV-2) (Khan et al., 2020). Apresenta perfil clínico extremamente variado e inespecífico, que pode variar de pessoas assintomáticas até as formas mais graves da doença com pneumonia, insuficiência respiratória e a morte (Habas et al., 2020). Este trabalho demonstra o perfil de profissionais de saúde, reagentes ou não, para SARS-CoV-2 em um hospital referência no Sudoeste do Paraná.

Um primeiro aspecto a ser destacado quanto ao perfil dos profissionais diz respeito ao gênero desses trabalhadores. A maioria dos profissionais da saúde nas instituições de saúde são do sexo feminino e com idade inferior a 40 anos de idade. Estudos demostram a prevalência do sexo feminino no ambiente hospitalar com idades entre 20 anos e 40 anos (Lahner et al., 2020; Rodrigues et al., 2020).

A presença feminina é maior nos serviços de saúde principalmente da enfermagem e como pode-se observar é uma população com menos de 40 anos (Gomes et al., 2020), o que sugere que está a menos de 10 anos no mercado. Segundo o Conselho Federal de Enfermagem (2020), com a pandemia da COVID-19, os profissionais de saúde, com maior idade e/ou 
comorbidade, que atuam diretamente com os pacientes, devem ser afastados. Isto levou a necessidade de contratação intensa dos profissionais de saúde. Diante disso, a inserção de profissionais mais jovens e com pouca experiência em hospitais (Gomes et al., 2020).

A taxa de diagnóstico reagente de profissionais reagentes para SARS-CoV-2, foi de 12,9\%, corroborando com esse resultado, na Espanha foi demonstrado que 13\% dos casos reagentes para o vírus são entre profissionais de saúde (Jones, 2020). Em contrapartida, em um Hospital de Roma, apenas 2,7\% dos profissionais de saúde tiveram o exame reagente (Lahner et al., 2020), assim como no estudo realizado com os profissionais de saúde de Portugal, onde apenas $2,11 \%$ foram reagentes para COVID-19 (Sousa-Uva, Sousa-Uva, \& Serranheira, 2021). Na Itália identificou-se um percentual de $20 \%$ dos profissionais reagentes para o vírus, dentre estes, com alguns óbitos entre enfermeiros (Remuzzi \& Remuzzi, 2020). Cabe destacar que nenhum profissional foi a óbito durante a execução dessa pesquisa.

Com relação a sintomas, este estudo demonstrou que a cefaleia (15,4\%), dor de garganta (13,3\%), mialgia $(12,2 \%)$, coriza $(11,3 \%)$ e tosse $(11,3 \%)$ foram os sintomas com maior frequência no momento da testagem dos profissionais de saúde. Os estudos desenvolvidos por Chu et al., (2020) demonstram esse mesmo perfil. O autor analisou 54 casos em uma equipe médica e também constatou que os sintomas (febre, tosse, diarreia, entre outros) ocorreram tanto em pacientes reagentes como não reagentes para COVID-19. Neste sentido, cabe a discussão se existe uma associação entre sintomas específicos em pacientes reagentes para SARS-CoV-2. No presente estudo observou-se uma associação entre a presença de sintomas e casos reagentes para o vírus, indicando contágio pela COVID-19. Portanto, pacientes com sintomas suspeitos da doença COVID-19 devem realizar a triagem e manter o isolamento social (Addams \& Walls, 2020).

Embora a febre não tenha sido um dos sintomas prevalentes mostrado por esse estudo, segundo a Organização PanAmericana de Saúde (2020) os principais sintomas da COVID-19 são febre, cansaço e tosse seca, que podem também ser sintomas semelhantes a uma gripe comum (Organização Mundial da Saúde, 2020c). O que explicaria a manifestação de sintomas por profissionais não reagentes para a COVID-19, que poderiam, durante a execução desta pesquisa, estar com outra patologia, como a gripe comum.

Estudo semelhante foi realizado por Lahner et al., (2020) em um Hospital de Roma, também dedicado ao atendimento à COVID-19. Na pesquisa, foram testados 2.057 profissionais de saúde, com prevalência dos sintomas: febre (34,7\%), ageusia $(34,7 \%)$, anosmia $(26,5 \%)$, tosse $(22,4 \%)$, astenia $(20,4 \%)$ e mialgia $(20,4 \%)$. Outro estudo, realizado em um Hospital Universitário de Wuhan, na China, envolvendo 158 pacientes reagentes para COVID-19, os principais sintomas foram: febre, fadiga e tosse seca (Wang et al., 2020). Na Coréia, ao avaliar 328 pacientes de dois hospitais, sintomáticos não graves, os sintomas mais frequentes foram tosse, escarro, dor de garganta e rinorreia (Uhm et al., 2020). Sintomas esses, semelhantes a alguns apresentados nesta pesquisa.

Dos casos reagentes 59,3\% ( $\mathrm{n}=48)$ não apresentaram sintomas. De forma similar, Rivett et al., (2020) mostraram que 57\% de reagentes para COVID-19 são assintomáticos/paucissintomáticos, ou seja, passaram a desenvolver alguns sintomas após algumas semanas do resultado. Índices bem mais baixos foram encontrados por Treibel et al., (2020), onde 27\% dos profissionais de saúde reagentes para COVID-19 permaneceram assintomáticos, semanas antes e também após a realização do teste. Outro estudo também neste sentido realizado por Mizumoto et al., (2020) relatam que bordo do navio de cruzeiro Diamond Princess, Yokohama, Japão, foi constatado um total de 634 pessoas com resultado positivo para Covid-19 entre 3.063 testes realizados em 20 de fevereiro de 2020, e destes 50,6\% foram de pessoas assintomáticas. Na China, em abril de 2020, as autoridades chinesas começaram a publicar dados demonstrando que a maioria das infecções por coronavírus são assintomáticas. A Comissão Nacional de Saúde da China divulgou que no dia $1^{\circ}$ de abril, que em 24 horas, obtiveram 166 casos positivos e destes 130 assintomáticos, ou seja 78\% da amostra, que pode ser uma importante fonte de contágio (Day, 2020). Esses resultados mostram a importância de pesquisas que procuram estabelecer a frequência/contaminação por 
COVID-19 em unidades de saúde, pois esses profissionais assintomáticos, se não tivessem sido testados, continuariam com suas rotinas diárias podendo disseminar ainda mais o vírus.

Mais da metade dos profissionais de saúde desempenham funções diretamente com o paciente. Destes, somente 12,9\%, o que equivale a 81 pessoas, trabalham com pacientes COVID-19, no entanto, apenas 12,3\% ( $\mathrm{n}=10)$, dos profissionais que trabalham diretamente com pacientes COVID-19, foram reagentes. Esses resultados demonstram que a maior parcela de contaminação ocorre em outras alas do hospital, 87,7\% (clínica geral, UTI geral, neonatal ou em outros setores) ou até mesmo fora das dependências do hospital. Corroborando com essa hipótese, de que os profissionais que atuam na unidade hospitalar que mais tiveram resultados reagentes para COVID-19 foram as categorias que não prestaram serviços aos pacientes Covid-19.

Além do ambiente hospitalar, que seria o local de trabalho, a contaminação por COVID-19 pode ocorrer, segundo a Organização Pan-Americana de Saúde em locais fechados, com ventilação inadequada, restaurantes, bares/boates e cultos (Organização Mundial da Saúde, 2020b). Estudos mostram perfil semelhante. Em Wuhan, na China, a infecção de 77,5\% dos profissionais de saúde ocorreu em enfermarias gerais, 17,5\% em pronto-socorro e apenas 5\% em UTI (Wang et al., 2020). Já na província de Hubei, na China, a contaminação ocorreu com maior frequência nos departamentos de emergência $(18,5 \%)$ (Chu et al., 2020). Também conforme o estudo realizado no Hospital Tongji, com foco na identificação da contaminação por COVID-19 nas equipes médicas, encontrou 54 pessoas atingidas pelo vírus. Desse quantitativo 72,2\% atuavam em enfermarias clínicas, 18,5\% na área de tecnologia médica e apenas 3,7\% estavam na emergência. Uma provável explicação é que dado as inúmeras manifestações clínicas atípicas da Covid-19 os pacientes podem ir para diferentes enfermarias (Chu et al., 2020). Em contrapartida, uma pesquisa desenvolvida no Hospital de Roma, mostrou que 50\% dos profissionais de saúde reagentes para COVID-19 tiveram contato direto com pacientes infectados (Lahner et al., 2020).

Cabe destacar que os profissionais que trabalham diretamente com pacientes COVID-19 utilizam EPIs que diminuem, ou quase eliminam, os riscos de contaminação, desde que utilizados de maneira correta. A utilização de EPIs, garante a continuidade da assistência, já que segura os trabalhadores do contágio ao COVID-19 (Cavalcante et al., 2021). De fato, o Ministério da Saúde (Ministério da Saúde, 2020c) relata uma menor frequência de contaminação em UTIs, devido a utilização de EPIs e aos protocolos de segurança.

Este estudo foi capaz de demonstrar a frequência de profissionais do HRSWAP reagentes para COVID-19, onde o maior número de contaminações não ocorreu em profissionais da saúde que atendem diretamente o paciente COVID-19, mas sim, nos que atuam em outras alas do hospital. Além disso, observou-se que mais da metade dos profissionais eram assintomáticos, ressaltando a importância da triagem.

Embora este estudo traga discussões sobre a contaminação de COVID-19 em profissionais de saúde, algumas limitações devem ser destacadas, como o fato de profissionais não reagentes para COVID-19 possuírem sintomas gripais. Deve-se considerar limitante também, a janela imunológica, uma vez que, os pacientes que tenham realizado o TR, tenham coletado antes do período recomendado para coleta que é a partir do $8^{\circ}$ dia de sintomas, não possuindo a quantidade de antígenos detectáveis pelo teste (Agência Nacional de Vigilância Sanitária, 2021). Ainda, em relação às amostras coletadas para o exame de RT-qPCR, estas devem ser realizadas o mais precocemente possível ainda quando o paciente está em sua fase aguda da infecção, preferencialmente do $3^{\circ}$ ao $7^{\circ}$ dia após o início dos sintomas, podendo ser realizada até no máximo até o $8^{\circ}$ dia após o início dos sintomas (Ministério da Saúde, 2020b).

\section{Considerações Finais}

Com este estudo, conclui-se que os profissionais, pela natureza de seu trabalho no atual contexto pandêmico, podem ser vetores de transmissão. Logo, é importante a realização de uma triagem constante, pois, como foi constatado, mais da metade dos casos confirmados eram profissionais assintomáticos os quais podem ampliar os surtos nas unidades de saúde, caso 
não sejam detectados precocemente. Logo, os profissionais de saúde devem sempre estar focados na promoção da saúde e prevenção de doenças. E os gestores devem assegurar que os equipamentos de proteção individual estejam apropriados para cada atividade, em quantidade suficiente e que sejam utilizados de maneira correta, e ainda disponibilizar treinamentos e capacitações frequentes aos trabalhadores de saúde com foco no uso eficiente das barreiras à exposição, reorganização dos fluxos operacionais e dos protocolos dos serviços.

Identificar e buscar compreender a introdução e a disseminação da COVID-19 nas unidades de saúde pelos profissionais permite fortalecer as políticas públicas para implantar protocolos de identificação e gerenciamento dos profissionais de saúde que estão expostos diretamente ou não aos pacientes com COVID-19. Tais esforços colaboram para a prevenção da transmissão do vírus dentro dos serviços de saúde, na proteção de outros profissionais, aos pacientes suscetíveis em ambientes de saúde e também para a sociedade.

A literatura científica ainda carece sobre a contaminação dos profissionais de saúde pelo vírus Sars-CoV-2, principalmente quando pretende-se observar o perfil brasileiro, o que demanda investigações futuras.

\section{Referências}

Anelli, F., Leoni, G., Monaco, R., Nume, C., Rossi, C. R., Marinoni, G., Spata, G., \& Piscitelli, P. (2020). Italian doctors call for protecting healthcare workers and boosting community surveillance during covid-19 outbreak. BMJ, 26(368), m1254.

Adams, G. J., \& Walls, M. R. (2020). Apoiando a força de trabalho da área da saúde durante a pandemia global de COVID-19. JAMA, 323(15), 1439-1440.

Agência Nacional de Vigilância Sanitária (2021). Nota técnica $n N^{o}$ 7/2021/SEI/GRECS/GGTES/DIRE1/ANVISA. Agência Nacional de Vigilância Sanitária.

Cavalvante, S. J. F., Nascimento, D. R. I., \& Cavalcante, S. M. R. (2021). EPIs e segurança dos trabalhadores de saúde durante a pandemia de COVID-19: Uma breve reflexão. Research, Society and Development, 10(7), e55010716809.

Chu, J., Yang, N., Wei, Y., Yue, H., Zhang, F., Zhao, J., He, L. \& Zhang, H. (2020). Clinical characteristics of 54 medical staff with COVID-19: A retrospective study in a single center in Wuhan. Journal of Medical.Virology, 92(7), 807-813.

Conselho Federal de Enfermagem (2020). Cofen vai à Justiça para preservar os profissionais integrantes do grupo de risco. http://www.cofen.gov.br/cofenvai-a-justica-para-preservar-profissionais-integrantes-dos-grupos-de-risco_79210.html

Day, M. (2020). Covid-19: four fifths of cases are asymptomatic, China figures indicate. BMJ, 369, m1375.

Gomes, P. M., Barbosa, D. J, Gomes, T. M. A., Souza, A. B. F. de, Paula, S. G. de, \& Espírito Santo, C. C. do. (2020). Perfil dos profissionais de enfermagem que estão atuando durante a pandemia do novo Coronavírus. Journal of Nursing and Health, 10, e20104026.

HRSWP. (2020). Hospital Regional do Sudoeste Walter Alberto Pecóits. Recursos Humanos. Impresso.

Habas, K., Nganwuchu, C., Shahzad, F., Gopalan, R., Haque, M., Rahman, A., Majumder, A. A., \& Nasim, T. (2020). Resolution of coronavirus disease 2019 (COVID-19). Expert Review Anti-Infective Therapy, 18(12), 1201-1211.

Jones, S. (2020). Spain: doctors struggle to cope as 514 die from coronavirus in a day. https://www.theguardian.com/world/2020/mar/24/spain-doctors-lackprotection-coronavirus-covid-19

Khan, M., Adil, F. S., Alkhathlan, Z. H., Tahir, N. M., Saif, S., Khan, M., \& Khan, T. S. (2020) Covid-19: A Global Challenge with Old History, Epidemiology and Progress So Far. Molecules, 26(1), 39.

Lahner, E., Dilaghi, E., Prestigiacomo, C., Alessio, G., Marcellini, L., Simmaco, M., Santino, I. \& Napoli, C. (2020). Prevalence of Sars-Cov-2 Infection in Health Workers (HWs) and Diagnostic Test Performance: The Experience of a Teaching Hospital in Central Italy. International Journal of Environmental Research and Public Health, 17(12), 4417.

Ministério da Saúde (2020a). Painel de casos da doença pelo coronavírus 2019 (COVID-19) no Brasil pelo Ministério da Saúde. Ministério da Saúde.

Ministério da Saúde (2020b). Guia de Vigilância Epidemiológica: Emergência de Saúde Pública de Importância Nacional pela Doença pelo Coronavírus 2019. Brasília: Ministério da Saúde.

Ministério da Saúde (2020c). Recomendações de proteção aos trabalhadores dos serviços de saúde no atendimento de COVID-19 e outras síndromes gripais. Brasília, Ministério da Saúde.

Mizumoto, K., Kagaya, K., Zarebski, A., \& Chowell, G. (2020). Estimating the asymptomatic proportion of coronavirus disease 2019 (COVID-19) cases on board the Diamond Princess cruise ship, Yokohama, Japan, 2020. EuroSurveillance, 25(10), 2000180.

Organização Mundial da Saúde (2020). Histórico da Pandemia. OMS. 
Research, Society and Development, v. 10, n. 9, e5310917662, 2021

(CC BY 4.0) | ISSN 2525-3409 | DOI: http://dx.doi.org/10.33448/rsd-v10i9.17662

Ornell, F., Schuch, B. J., Sordi, O. A., \& Kessler, P. H. F. (2020). "Pandemic fear" and COVID-19: mental health burden and strategies. Brazilian Journal of Psychiatry, 42(3), 232-235.

Pereira, S. A., Shitsuka, M. D., Parreira, J. F., \& Shitsuka, R. (2018). Metodologia da pesquisa científica. https://repositorio.ufsm.br/bitstream/handle/1/158 24/Lic_Computacao_Metodologia-Pesquisa-Cientifica.pdf?sequence=1

Remuzzi, A., \& Remuzzi, G. (2020). COVID-19 e Itália: o que vem a seguir? Lanceta, 395(10231), 1225-1228.

Rodrigues, M. C., Domingues, E. T., Mendes, F. B., Lopes, P. A. I., Magalhães, D. O. C. e, Corrêa, G. M. M. P., Vasconcelos, F. B. \& Ribeiro, C. C. L. (2020). Perfil e condições laborais da enfermagem no enfrentamento da Covid-19. International Journal of Development Research, 10(9), 40175-40179.

Rivett, L., Sridhar, S., Sparkes, D., Routledge, M., Jones, K. N., Forrest, S., Young, J. \& Weekes, P. M. (2020). Screening of healthcare workers for SARSCoV-2 highlights the role of asymptomatic carriage in COVID-19 transmission. eLife, 9, e58728.

Shah, K., Kamrai, D., Mekala, H., Mann, B., Desai, K., \& Patel, S. R. (2020). Focus on mental health during the coronavirus (COVID19) pandemic: applying learnings from the past outbreaks. Cureus, 12(3), e7405.

Sousa-Uva, M., Sousa-Uva, A., \& Serranheira, F. (2021). Prevalência de COVID-19 em profissionais de saúde e riscos profissionais de natureza psicossocial. Revista Brasileira de Medicina do Trabalho, 19(1), 73-81.

Treibel, T. A., Manisty, C., Burton, M., McKnight, A., Lambourne, J., Augusto, B. J., Couto-Parada, X., \& Moon, C. J. (2020). COVID-19: PCR screening of asymptomatic health-care workers at London hospital. Lancet, 395, 1608-1610.

Uhm, J. S., Ahn, Y. J., Hyun, J., Sohn, Y., Kim, H. J., Jeong, J., Ku, S. N. \& Park, S. Y. (2020). Patterns of viral clearance in the natural course of asymptomatic COVID-19: Comparison with symptomatic non-severe COVID-19. Elsevier Public Health Emergency Collection, 99, 279-285.

Wang, D., Hu, B., Hu, C., Zhu, F., Liu, X., Zhang, J. Wang, B. \& Peng, Z. (2020). Clinical Characteristics of 138 Hospitalized Patients With 2019 Novel Coranavirus-Infected Pneumonia in Wuhan, China. JAMA, 323(11), 1061-1069. 